\title{
ON CHARACTERIZATION OF RIEMANNIAN MANIFOLDS BY GROWTH OF TUBULAR NEIGHBORHOODS ${ }^{1}$
}

\author{
NATHANIEL GROSSMAN
}

\begin{abstract}
If the area function of the tubular neighborhoods of a compact submanifold of a Riemannian manifold satisfies a certain linear differential inequality, then the codimension of the submanifold is at most the order of that inequality.
\end{abstract}

1. Let $N$ be a Riemannian manifold of dimension $n \geqq 2$ and let $M$ be a compact orientable submanifold of dimension $m$ embedded in $N$. (All manifolds, maps, etc. are supposed smooth.) For $s>0$, let $M_{s}$ denote the set of points lying on geodesics normal to $M$ and at arc length $s$ from $M$. For sufficiently small $s, M_{s}$ is a smooth hypersurface in $N$. We denote by $\mathscr{A}(s)$ the area of $M_{s}$. H. Wu [2] derived an elegant formula for $\mathscr{A}^{\prime \prime}(s)$ and used it to characterize the Euclidean plane amongst Riemannian manifolds. Later he and R. A. Holzsager [1] proved the following more encompassing characterization:

A Riemannian manifold has the property that the growth function $\mathscr{A}$ of each one of its compact hypersurfaces satisfies the linear differential equation

$$
\mathscr{A}^{\prime \prime}(s)+c \mathscr{A}(s)=0
$$

(where $c$ is a fixed constant) if and only if it is a two-dimensional Riemannian manifold of constant curvature equal to $c$.

In this note we obtain a formula for $\mathscr{A}^{\prime \prime}(s)$ and indeed for all the derivatives of $\mathscr{A}$, valid for submanifolds $M$ of any dimension. Our method yields $\mathscr{A}^{\prime \prime}$ in a simpler, more mechanical fashion than does Wu's. We can easily reprove the theorem of Holzsager and Wu. We also obtain an extension of their theorem to the case when $\mathscr{A}$ satisfies a linear differential inequality of higher order with "nice" coefficients. We show the order of this differential inequality is an upper bound for the codimensions of the submanifolds involved.

Received by the editors July 9, 1971.

AMS 1970 subject classifications. Primary 53B20, 53B25; Secondary 53C20, 53C40.

Key words and phrases. Riemannian, tubular neighborhood, area function, differential inequality, constant curvature, Cartan calculus.

1 This research was partially supported by NSF Grant GP-11476.

(c) American Mathematical Society 1972 
2. Let $M$ be a fixed compact orientable submanifold of $N$ with an embedded tubular neighborhood $U$ in $N$. Let $U^{*}=U-N$. Our arguments are local, so we may suppose $N$ to be oriented. Choose a positively oriented (local) orthonormal frame field $e_{1}, \cdots, e_{n}$ on $U^{*}$, supposed adapted so $\boldsymbol{e}_{1}$ is the tangent vector of geodesics leaving $M$ normally, while each $\boldsymbol{e}_{i}$ $(2 \leqq i \leqq n)$ is parallel along such a geodesic. Let $\omega_{1}, \cdots, \omega_{n}$ be the dual coframe and let $\omega_{i j}(1 \leqq i, j \leqq n)$ be the connection 1-forms of the Riemannian structure of $N$ as restricted to $U^{*}$. The forms $\omega_{i}, \omega_{i j}$ satisfy the Cartan structural equations

and

$$
d \omega_{i}=\sum_{j=1}^{n} \omega_{i j} \wedge \omega_{j}
$$

$$
d \omega_{i j}=\sum_{k=1}^{n} \omega_{i k} \wedge \omega_{k j}+\Omega_{i j}
$$

where $\Omega_{i j}$ is the curvature 2 -form.

For sufficiently small $s>0, M_{s} \subset U^{*}$ and is a hypersurface with normal field $e_{1}$ so there are functions $h_{i j}=h_{j i}(2 \leqq i, j \leqq n)$ on $U^{*}$ with

$$
\omega_{1 i}=\sum_{j=2}^{n} h_{i j} \omega_{j} .
$$

The mean curvature is given at $p \in M_{s}$ by

$$
H(p)=\frac{1}{n-1} \sum_{j=2}^{n} h_{j j}(p) .
$$

(Note that the signs have been chosen so that $M_{1}$ has $H=1$ when $M$ is the origin in the Euclidean plane.) Finally, the function $\mathscr{A}(s)$ is given by

$$
\mathscr{A}(s)=\int_{M_{s}} \omega_{2} \wedge \cdots \wedge \omega_{n}
$$

We use Stokes' theorem on the closed set $M_{s+\delta, s}$ included between $M_{s}$ and $M_{s+\delta}(\delta>0)$. We then have

$$
\mathscr{A}(s+\delta)-\mathscr{A}(s)=\int_{M_{s+\delta, s}} d\left(\omega_{2} \wedge \cdots \wedge \omega_{n}\right) .
$$

Using the first Cartan structural equation, we calculate

Hence

$$
d\left(\omega_{2} \wedge \cdots \wedge \omega_{n}\right)=(n-1) H \omega_{1} \wedge \cdots \wedge \omega_{n} .
$$

$$
\mathscr{A}(s+\delta)-\mathscr{A}(s)=\int_{M_{s+\delta, s}}(n-1) H \omega_{1} \wedge \cdots \wedge \omega_{n}
$$


Keeping in mind the geometrical meaning of $\omega_{1}$, we divide by $\delta$ and let $\delta \rightarrow 0$ to obtain

$$
\mathscr{A}^{\prime}(s)=\int_{M_{s}}(n-1) H \omega_{2} \wedge \cdots \wedge \omega_{n} .
$$

In order to calculate higher derivatives of $\mathscr{A}$, it will be convenient to use this notation: if $F$ is a smooth function on $U^{*}$, then

$$
d F=\sum_{j=1}^{n} F_{j} \omega_{j}
$$

Again applying Stokes' theorem, we have

It follows easily that

$$
\mathscr{A}^{\prime}(s+\delta)-\mathscr{A}^{\prime}(s)=\int_{M_{s+\delta, s}}(n-1) d\left(H \omega_{2} \wedge \cdots \wedge \omega_{n}\right) .
$$

$$
\mathscr{A}^{\prime \prime}(s)=\int_{M_{s}}(n-1)\left\{H_{1}+(n-1) H^{2}\right\} \omega_{2} \wedge \cdots \wedge \omega_{n} .
$$

There is a general formula:

$$
\mathscr{A}^{(k)}(s)=\int_{M_{s}} I_{k} \omega_{2} \wedge \cdots \wedge \omega_{n} .
$$

The functions $I_{k}$ satisfy the recurrence relation

$$
I_{k+1}=I_{k, 1}+(n-1) H I_{k} \text {. }
$$

We will not attempt to give an explicit formula for $I_{k}$ in terms of $H$ and its derivatives.

3. Now we will extend the theorem of Holzsager and $\mathrm{Wu}$. The following estimate generalizes the lemma of $\mathrm{Wu}$ [2].

LEMMA 1. As $s \rightarrow 0, H \sim(n-m-1) /(n-1) \cdot 1 / s$. This asymptotic relation is uniform from one normal geodesic to another and may be differentiated as often as desired.

(Here we use the asymptotic symbol $\sim$ in the usual sense: $f \sim g$ means $f(s) / g(s) \rightarrow 1$ as $s \rightarrow 0$.)

Verification of the asymptotic relation of Lemma 1 along a given normal geodesic proceeds from an elementary calculation using the first and second Cartan structural equations. We obtain relations expressing the successive derivatives of each $h_{i i}$ in terms of the lower-order radial derivatives of $h_{i i}$ as well as the Riemannian curvature and its derivatives. Using the smoothness of the data, we establish inductively that each radial derivative of $h_{i i}$ has the proper order of infinity as $s \rightarrow 0$. Knowing this, we can use the 
uniqueness of asymptotic expansion to calculate the asymptotic behavior of the derivatives of $H$ as $s \rightarrow 0$. The compactness of $M$ allows the conclusion that these asymptotic estimates are uniform over $M$ from one normal geodesic to another. The perturbation techniques used are by now standard in global analysis.

Using the recurrence relation for $I_{k}$ and Lemma 1 , we can prove an estimate for $I_{k}$ by induction.

LEMMA 2. As $s \rightarrow 0$,

$$
I_{k} \sim(n-m-1) \cdots(n-m-k) s^{-k},
$$

and this estimate is uniform from one normal geodesic to another.

Here is our main result.

THEOREM. Let $k$ be a positive integer and fix $m \leqq n-2$. Suppose for each compact $M$ of dimension $m$ there are bounded continuous functions $c_{1}^{M}, \cdots$, $c_{k}^{M}$ such that the area function $\mathscr{A}$ of $M$ satisfies the differential inequality

$$
\mathscr{A}^{(k)}(s)+\sum_{j=1}^{k} c_{j}^{M}(s) \mathscr{A}^{(k-j)}(s) \leqq 0
$$

for sufficiently small $s>0$. Then $n-m \leqq k$.

The proof is based upon Lemma 2. We have

$$
\mathscr{A}^{(j)}(s)=\int_{M_{s}} I_{j} \omega_{2} \wedge \cdots \wedge \omega_{n}
$$

whence the differential inequality hypothesized implies

$$
\int_{M_{s}}\left\{I_{k}+\sum_{j=1}^{k} c_{j}^{M}(s) I_{k-j}\right\} \omega_{2} \wedge \cdots \wedge \omega_{n} \leqq 0 .
$$

By Lemma 2, the function in curly brackets has the uniform asymptotic expression

$$
\{\cdots\}=(n-m-1) \cdots(n-m-k) s^{-k}+o\left(s^{-k}\right)
$$

as $s \rightarrow 0$. If $n-m>k,\{\cdots\}>0$ for sufficiently small $s>0$, so

$$
\int_{M_{s}}\{\cdots\} \omega_{2} \wedge \cdots \wedge \omega_{n}>0 .
$$

This contradiction shows $n-m \leqq k$, as was claimed.

4. We show how to obtain the theorem of Holzsager and Wu. Suppose

$$
\mathscr{A}^{\prime \prime}(s)+c \mathscr{A}(s)=0
$$


for some constant $c$. We apply our theorem with submanifolds $M$ consisting of single points, so $m=0$. We conclude $n=2$. Calculating $I_{2}$ explicitly, we find

$$
\mathscr{A}^{\prime \prime}(s)+c \mathscr{A}(s)=\int_{M_{s}}\left(H_{1}+H^{2}+c\right) \omega_{2}
$$

In our case, $M_{s}$ is a curve. In terms of the adapted coframe field, $\omega_{12}=$ $H \omega_{2}$. The Gauss curvature function $K$ satisfies

$$
d \omega_{12}=-K \omega_{1} \wedge \omega_{2}
$$

from which we obtain $H_{1}+H^{2}=-K$. Therefore,

$$
\int_{M_{s}}(c-K) \omega_{2}=0
$$

Since $M$ and $s$ are arbitrary, $K \equiv c$ on $N$.

\section{BIBLIOGRAPHY}

1. R. A. Holzsager and $\mathrm{H}$. Wu, A characterization of two-dimensional Riemannian manifolds of constant curvature, Michigan Math. J. 17 (1970), 297-299.

2. H. Wu, A characteristic property of the euclidean plane, Michigan Math. J. 16 (1969), 141-148. MR 39 \#7544.

Department of Mathematics, University of California, los Angeles, CaliFORNIA 90024 